\title{
Corrections to the paper
}

\author{
"On Thermodynamics \\ and Elastic-Plastic Materials" \\ Bernard D. Coleman \& David R. Owen
}

Volume 59, 25-51 (1975)

The relation (5.5) in Remark 5.1 on page 42 should be

$$
\left|\lambda_{e}^{\prime \prime}(\tau)-\lambda_{e}^{\prime}(\tau)\right|+\left|\lambda_{p}^{\prime \prime}(\tau)-\lambda_{p}^{\prime}(\tau)\right| \leqq\left|\lambda_{e}^{\prime \prime}(0)-\lambda_{e}^{\prime}(0)\right|+\left|\lambda_{p}^{\prime \prime}(0)-\lambda_{p}^{\prime}(0)\right| .
$$

This change does not affect the validity of any of the subsequent results of the paper. However, the symbol $\|\cdot\|$ in the proof of Theorem 5.1 should be interpreted as the norm on $\mathbb{R}^{2}$ defined by

$$
\|(x, y)\|=|x|+|y|,
$$

rather than the Euclidean norm. 